\title{
Rethinking Interest in Studies of Interactive Information Retrieval
}

\author{
Luanne Sinnamon \\ School of Information \\ University of British Columbia \\ Vancouver B.C. Canada \\ luanne.sinnamon@ubc.ca \\ Samuel Dodson \\ School of Information \\ University of British Columbia \\ Vancouver B.C. Canada \\ dodsons@mail.ubc.ca
}

\author{
Limor Tamim \\ School of Information \\ University of British Columbia \\ Vancouver B.C. Canada \\ limor.tamim@gmail.com \\ Heather L. O’Brien \\ School of Information \\ University of British Columbia \\ Vancouver B.C. Canada \\ h.obrien@ubc.ca
}

\begin{abstract}
Interest characterizes a cognitive-emotional relationship between people and information and is a key construct in human information interaction. As a motivational variable, interest has been widely studied in psychology and education, but has received less consistent and theory-driven attention in the field of interactive information retrieval (IIR). In this perspective paper we examine the role of interest and review how it has been studied and operationalized in IIR research. We draw upon a survey of 58 research studies that have manipulated, controlled or measured searcher interest in some way. The intent of the paper is to raise the profile of interest as a user-centred variable in IIR and to advocate for more conceptual and methodological consistency in future studies to better evaluate the impact of interest in information search.
\end{abstract}

\section{CCS CONCEPTS}

-Information systems $\sim$ Information retrieval Users and interactive retrieval $\bullet$ Human-centered computing $\sim$ Human computer interaction (HCI) $\sim \mathrm{HCI}$ design and evaluation methods $\sim$ User studies

\section{KEYWORDS}

Interest, research methods, user studies, assigned search tasks

\section{ACM Reference format:}

Luanne Sinnamon, Limor Tamim, Samuel Dodson, Heather L. O’Brien. 2021. Rethinking interest in studies of interactive information retrieval. In Proceedings of ACM SIGIR Conference on Human Information Interaction and Retrieval (CHIIR '21). ACM, New York, NY, USA. 11 pages.

https://doi.org/10.1145/3406522.3446031

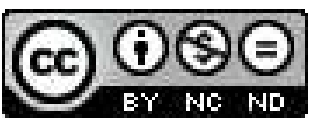

This work is licensed under a Creative Commons Attribution-NonCommercialNoDerivs International 4.0 License.

CHIIR '21, March 14-19, 2021, Canberra, Australia.

(C) 2021 Copyright is held by the owner/author(s).

ACM ISBN 978-1-4503-8055-3/21/03. https://doi.org/10.1145/3406522.3446031

\section{Introduction}

Interest is a widely acknowledged motivational factor in information seeking and knowledge construction. Interest drives information seekers to persist and surpass comfortable knowledge limits, moving them towards new discoveries and offering intrinsic intellectual and emotional rewards [30,58,72]. Interest, in this sense, has been studied extensively in the domains of psychology and education, giving rise to a robust theoretical and empirical foundation. To date, it has played a relatively minor role in studies of interactive information retrieval (IIR), where treatments of interest have relied primarily on common-place notions of topical interest that influence search behaviour and preferences. However, with increased attention being paid to the human information interaction (HII) processes that occur during search and the associated user experience, interest has been identified as a key factor in user engagement [5,20,56]; the information experience [74]; and exploration and learning [80]. Through this research, more sophisticated notions of interest, drawing upon seminal research in educational psychology $[30,72]$, have been introduced to the IIR community. However, it is still the case that definitions and operationalizations of interest are inconsistent and, as noted by Edwards and Kelly [19], guidance on how to manipulate interest in experimental research is lacking. This is true of other disciplines as well: a systematic review of biology education found that $67 \%$ of the works reviewed measured interest, but only $37 \%$ referenced theory and $26 \%$ defined the term [64].

As IIR research extends towards ever broader contexts and applications of search, it is important that we develop shared perspectives on, and methods for, the control and manipulation of key variables, including interest. Over the past decade, research has examined highly interactive search contexts, focusing on the experiential, social and emotional aspects of search [3,44,51,74]; exploratory search [80,82]; casual-leisure search [21]; creativedesign driven search [84]; meaning-making [65]; and searching as learning [24,27]. In all these contexts, the effort, emotional experience, and personal development of the searcher over the course of the search session is paramount, much like educational 
settings. However, we do not have a complete picture of how to design search systems and information environments that foster and support the active involvement of searchers in that process, which is a key goal for interactive systems [5,74]. We do know that interest functions as an intrinsic motivational factor in information seeking and learning [30,72], and as such, is worthy of greater attention.

The goal of this perspective paper is to improve the clarity and consistency of treatments of interest in IIR, such that we can design effective studies and reach a deeper understanding of the role that interest plays in searching and associated outcomes. While acknowledging that notions of interest underpin personalization and recommender systems, we have left that work largely outside the scope of this paper, which is a limitation necessitated by the breadth of this topic. We begin by defining and reviewing well-known models of interest from educational psychology to establish a common vocabulary and make the case for the importance of interest as an IIR variable (Section 2). We then examine how interest has been viewed and studied to date in IIR in relation to core concepts: information needs, relevance, and the user experience (Section 3). In Section 4 we review approaches to studying interest in IIR, focusing on the use of assigned search tasks (ASTs) and methods of measuring interest. Based on this review, we identify some issues involved in the control, manipulation and measurement of interest and make some recommendations for future steps (Section 5), before concluding the paper (Section 6).

\section{The educational psychology view of interest}

\subsection{What is interest?}

To inform the study of interest as a factor in IIR, we draw upon foundational work in educational psychology. Interest is defined as a construct that characterizes a relationship between an individual and semantic content. Hidi and Renniger [30] distinguish between two separate interest phenomena: a psychoemotional state arising through interaction between an individual and semantic content, and a disposition towards particular types of information items. The first, known as state interest, is the individual's experienced state during interaction with specific content, comprising affective and cognitive components and typically characterized by increased attention, effort, concentration, and positive affect [61]. The second phenomenon is an idiosyncratic, internally driven, and relatively enduring predisposition to reengage with some types of content, referred to as individual interest [30,61]. Individual interest functions as a motivational variable that influences predispositions towards content. Unlike state interest, which is an in-the-moment experience, these predispositions persist over time, and, when well developed, they may be perceived by an individual to constitute part of their identity and self-definition [40]. Hidi and Renninger [30] proposed a four-phase model of the process of individual interest development (Section 2.2). Another important term defined by Hidi and Renninger [30] is situational interest, which is a type of state interest: an in-the-moment context-dependent, motivational experience of interacting with specific content, triggered by extrinsic factors, e.g., environmental conditions or objects [62]. Silvia [72] highlights the motivational effects of interest, noting its important role in supporting learning and developing knowledge and expertise. In contrast to Hidi and Renninger [30] he conceptualizes interest as an emotion, which entails instinctive and automatic responses to stimuli. Specifically, he positions interest as one of the knowledge emotions, a family of emotions that support exploration, reflection and learning [72]. Silvia proposes an appraisal theory to explain the evaluation processes that activate the emotional response of interest [72].

While these conceptualizations of interest differ, they also converge in characterizing it as a source of motivation to engage with content that features both cognitive and affective dimensions. In an IIR scenario, a searcher's state of interest may arise from individual interest, meaning the predisposition to engage with a topic or type of content, or it could be triggered by extrinsic factors including the search task or viewing search results with certain properties, such as novelty or complexity (i.e., situational interest). According to Renniger and Hidi [61] state interest is experienced the same, regardless of whether it stems from intrinsic predispositions or extrinsic factors. In search, individual interests could be expressed by carrying out extended and repeated search sessions in particular domains of interest, such as the environment, cooking or politics, and therefore individual interests are typically associated with prior knowledge. Individual interest in a topic or domain can increase the likelihood of entering into a state of interest while undertaking an activity such as searching, given the predisposition towards related content, but it does not necessarily lead to state interest, as other aspects of the situation (e.g., content features mood of the searcher, available time) may have an impact.

\subsection{Theories and models of interest}

Beyond defining the construct, theories of interest from educational psychology offer insights into its role in information seeking and learning, which are applicable to IIR.

\subsubsection{Silvia's appraisal theory of interest}

Silvia's appraisal theory of interest addresses the question of how people become interested. In psychology, appraisal theory claims that emotions are an instinctive response to the evaluation (or appraisal) of specific situations or events. For example, evaluating a situation as dangerous is likely to elicit fear. Multiple people may appraise the same situation differently and therefore have diverse emotional responses [71]. Silvia's theory specifies two appraisal processes involved in triggering interest [72]:

1. Novelty-Complexity: appraisal of the perceived complexity and ease of processing an event; the extent to which it is new and unexpected.

2. Comprehensibility: appraisal of one's coping-potential; the extent to which one has the skills, knowledge, and resources to deal with an event.

The theory claims that for something to trigger an interest response it must be appraised as new, sufficiently complex, and comprehensible by that individual. Interest motivates learning: a 
positive emotional response prompts interaction with content, which leads to knowledge construction. Knowledge construction sustains interest: more advanced and complex material on a topic will become comprehensible over time, and therefore interesting. This theory has implications for the selection and presentation of content in IIR systems, and several researchers have adopted Silvia's model in their work, notably van der Sluis et al. [74].

\subsubsection{The four-phase model of interest development}

Hidi and Renninger [30] propose a process model of the development of a person's interest from indifference to welldeveloped individual interest. The model considers interest to be a motivational variable in an extended learning process of days, weeks, or years, which includes affective and cognitive components. The identified phases of the model are outlined in Figure 1. Phases are sequential and interest develops with the transition from one phase to the next; however, interest may stagnate or regress to previous phases, depending on the context and the level of inherent challenge, reward and support. Thus, situational interest may or may not lead to well-developed individual interest. Each phase is characterized by varying amounts of affect, knowledge, and perception of content value. Knowledge is assumed to increase over the phases, and the length and nature of a given phase are influenced by individual experience, temperament, and genetic predisposition. The model articulates the relationship between individual and situational forms of interest and provides a theoretical basis for examining the role of interest in longitudinal, session-based searching for learning, casual leisure or other interest driven activities. To our knowledge, it has received limited attention in IIR research.

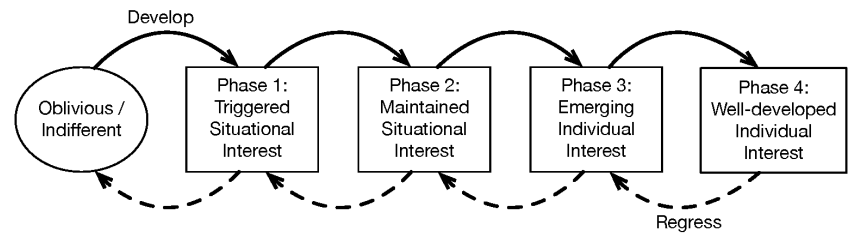

Figure 1. Four-Phase Model of Interest Development, adapted from [30]

\subsubsection{Interest and motivation}

Motivation is considered essential for learning, and there is a rich body of work on motivational theory (e.g., [61,68]). Extrinsic, goaloriented motivations for learning focus on the thoughts, feelings and beliefs of learners and information seekers [68] and align with task-based approaches to searching. Influential goal-oriented theories of motivation include self-efficacy, which considers a person's confidence in their ability to accomplish goals [7], and task value, or achievement theory, which consider the valuation of the goal and expectation of success [81]. Self-determination theory [67] is a broad framework that views both implicit and explicit factors contributing to a person's experience of autonomy, competence and relatedness as key to motivation.

As an intrinsic motivational variable, interest stands out in several ways [30]: unlike extrinsic motivations that are framed cognitively around beliefs, goals and expectations, interest combines affective and cognitive components. It arises from the interaction between a person and an information object, rather than functioning as a general disposition [30,38]. Further, the interest mechanism is presented as a deeply rooted biological and evolutionary driver associated with seeking behaviour in mammals [30]. As such, it may offer fruitful paths of inquiry in connection with familiar IIR models of berry-picking [8] and information foraging [59]. Silvia [72] helps to articulate the specific contribution of interest through comparison with happiness, describing how the two emotions serve opposite functions: both are motivational, but happiness encourages us to focus on familiar and past experiences that have been rewarding, interest pushes us to explore novel and complex phenomena. As a source of intrinsic motivation, interest serves as a counterweight to anxiety triggered by uncertainty, and it has a significant role in intellectual discovery and the growth of knowledge and expertise [72].

\subsection{Why is interest important?}

Empirical research on interest in educational psychology has identified impacts on many aspects of the learning experience that are relevant to IIR. While it is not possible to survey all of the prior research on this topic, Table 1 provides a limited summary of observed positive relationships with interest, categorized into five types of outcomes: learning and comprehension, cognitive and perceptual outcomes, effort, user experience, and academic performance.

Table 1: Summary of outcomes influenced by interest

Learning and Comprehension

- Reading comprehension, inferencing [30]

- Integration of information with prior knowledge [36]

- Knowledge acquisition [20,62,72]

Cognitive and Perceptual Outcomes

- Recognition and recall [63]

- Cognitive performance $[2,30]$

- $\quad$ Focused attention $[30,47,62]$

Effort

- Persistence $[26,30,62,72,77]$

- $\quad$ Effort $[30,33]$

- Duration of engagement [72]

User Experience

- In-the-moment motivation to explore [39]

- Positive emotions [30]

- Perception of ease [26]

Academic Performance

- Grades [72]

- Academic achievement [39]

A positive relationship between interest and learning, in the form of reading comprehension, knowledge acquisition and knowledge construction has been noted in numerous studies. The mechanisms for these effects on learning are observed in studies showing increased cognitive performance including focused attention, recognition, and recall; and increased effort in the form 


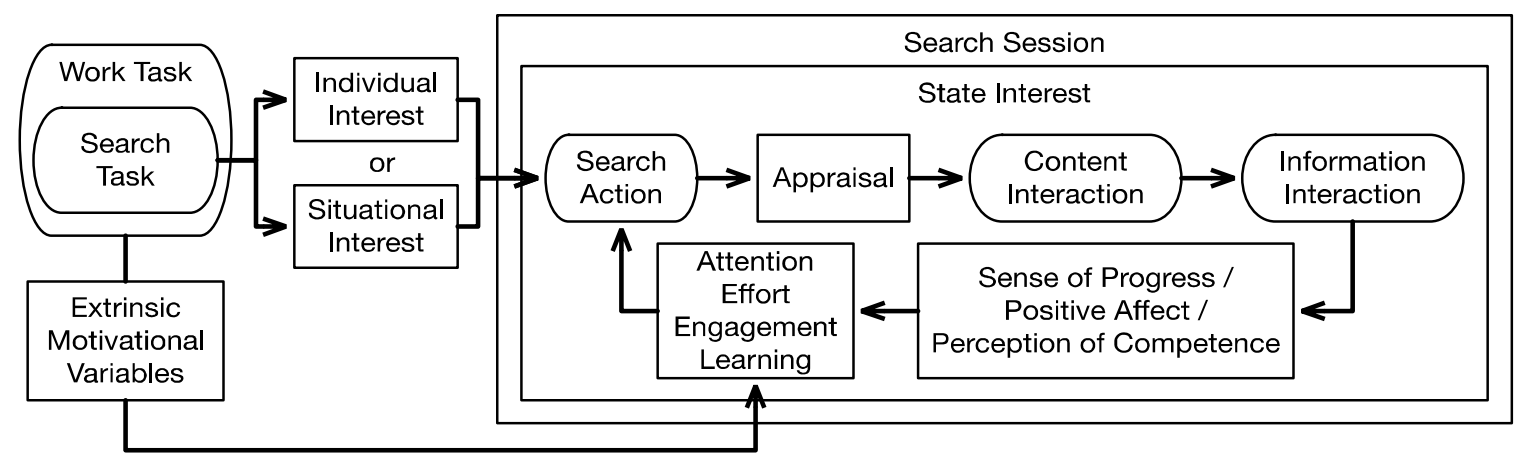

Figure 2. Conceptual model of the role of interest in IIR

of persistence and time on task. Interest influences the user experience by prompting positive affect, feelings of ease and willingness to explore, and increasing the likelihood of experiencing engagement and flow. In academic environments, interest has been shown to influence grades and performance.

\subsection{Summary}

We have presented a brief overview of interest drawn from educational psychology, a cognate field to IIR, in which interest has been the focus of substantial research. Drawing upon this prior work, we summarize the core concepts and represent them in relation to the search process. Figure 2 illustrates a search session in which state interest is activated by individual interests or situational interest. We note that his scenario does not represent all searches, as some are driven purely by extrinsic motivation. Working from the left of the diagram, a search task arises from a work task context, which may include daily life tasks or individual interests $[16,32]$. As such the task may be aligned with a searchers' individual interests or it may trigger situational interest, or both. In any given search session, extrinsic motivational factors may also be at play: notably, the task content itself may trigger a desire for goal achievement or impact the searcher's confidence in their capability to carry out the search. When state interest occurs, it functions as a motivator during the search session. Appraisal mechanisms drive content selection and information interaction, and an ongoing state of interest attracts the searcher to content and positively influences attention, effort, and engagement. This leads to learning and a sense of progress that sustains or increases the searcher's interest and motivation to search.

Abstracted in this way, the role and importance of interest in search is clear; however, it is essential to note that the impact of interest can be subtle and difficult to isolate and measure in user studies, where many contextual factors influence the searcher and the situation. This summary applies approaches to interest from educational psychology $t$ the search process; Section 3 will review how interest has been conceptualized and studied in IIR to date.

\section{Investigations of interest in IIR research}

From its origins, information retrieval has been framed instrumentally, focusing on retrieving information relevant to articulated information needs in response to questions or problems. The users of early systems were experts in their fields, and the ability to make use of the retrieved information was assumed. In this context, the users' interest in the material was considered of little consequence relative to its topical relevance and usefulness. However, the context for search has changed dramatically over the last two decades and information systems supporting activities such as exploration, discovery and learning are now commonplace. Given what is known about interest, there is little doubt that it is a variable of importance in a range of search scenarios, including:

- Goal-oriented searches prompted by individual interests, such as serious or casual leisure pursuits [21] and meaning-making [65], which may prompt multi-session searching over extended periods;

- Searching and browsing driven by situational interest in nontask domains, such as news [5,55,78]; entertainment [21]; and fiction reading [57];

- Exploratory and problem-solving searching in which learning is a goal and effort and persistence are required for success $[14,27,80,82]$.

Nevertheless, in the context of IIR research, the role of interest as a motivational variable has been understudied and, where interest has been considered, it has been left undefined, with distinctions between dispositions and state interest largely ignored. For example, in the faceted classification of search tasks proposed by $\mathrm{Li}$ and Belkin [42], interest is not among the variables representing user perception of tasks. "Intrinsic interest" does appear in the more extensive list of task characteristics identified by Kim and Soergel [35]; however, this model has been less influential. Overall, interest has received little attention in taskbased search studies, with some recent exceptions [e.g., 19], but it has emerged as the subject of study in research on the emotional and experiential aspects of search. In order to situate the notion of interest within the IIR domain, the following subsections review how interest has been construed in relation to three key concepts: information needs, relevance, and user experience.

\subsection{Interest and information needs}

Information needs in the form of cognitive uncertainties or gaps in knowledge have been viewed as primary catalysts for information 
searching, with more recent work focusing on task goals and attributes as drivers of search [22]. While it is a commonplace notion that people often search for information out of interest this is not reflected in most information seeking and searching models. An exception is the work of Ingwersen and Jarvelin [32], which identifies "life-tasks or interests" as a source of information needs, which then establish the "cognitive space" of the searcher with respect to decisions of relevance and usefulness. This view has informed empirical research. In a study drawing upon participants' "real needs," Borlund and Pharo [13] found that information needs arose from participants' work, studies, and personal interests. Similarly, Savolainen and Kari [70] conducted a study of web searching using participant-chosen topics related to selfdevelopment, e.g., hobbies. They hypothesized that searching would be motivated by "an underlying task at hand or an interest in some issue that gives rise to the current search task" (p. 687). These perspectives are consistent with cognitive framings of information needs, as personal interests are treated as a context out of which needs, questions, and uncertainties may arise, rather than a set of dispositions or an affective state, as represented in Figure 2.

An alternate treatment of interest as the motivation for search can be found in casual leisure, where hedonic (i.e., pleasureoriented) needs may take primacy over epistemic needs $[21,80]$. For example, a study of searching for entertainment found that needs were largely unspecified, with searchers seeking "interesting, sophisticated or challenging" content [21] and that participants' search goals were often directed towards achieving a certain kind of mood or emotional state. Similarly, some studies of browsing have focused on interest as a driver of search and selection [78]. In this framing, achieving state interest, as represented in Figure 2, may take the place of cognitive information needs as the primary motivation for certain types of searches.

With respect to information needs, the close association between interest and curiosity is worth noting, particularly in browsing and exploratory search scenarios. While the two are frequently conflated in IIR research (e.g., [14,70,78]), they have distinct features. Pekrun [58] considers curiosity to be a special case of state interest in which a person recognizes an information gap and sees the possibility and potential reward for filling the gap [58]. Once filled, curiosity is satisfied, while interest, which is not predicated on a specific need or gap in knowledge, may persist. Curiosity has a stronger cognitive basis than interest and a clearer alignment with traditional notions of information needs, although both are motivational variables related to knowledge seeking and affective states [58,72].

\subsection{Interest and relevance}

As with information needs, the relationship between relevance and interest is not well defined. Many published papers in IIR casually use the term "interesting" as a stand-in for relevance, or to refer more generally to documents that a searcher selects. On the other hand, interest can be viewed as independent from relevance, with relevance indicating congruence between the information need and content, and interest representing an idiosyncratic personal attraction to content. A compelling example of this conflict with respect to situational interest is Bowler's [14] study of adolescents conducting research for an assigned essay who struggled to "ignore powerful feelings of interest" (p. 1339) in content they encountered in order to stay on task by viewing relevant, but less interesting, content. It follows that, in cases where searches are guided purely by interest [e.g., 78], relevance and interest judgements are likely to be aligned, but in assigned tasks, they may diverge significantly.

Some researchers have placed interest within frameworks of relevance. For example, a study of TREC assessors found that those who considered TREC topics to be interesting and had prior knowledge of them were more likely to judge retrieved documents as relevant [66]. These results suggest that individual interest may influence relevance judgements, perhaps through the increased ability to see connections with the topic. State interest can be viewed as a form of affective relevance [6,74]. Cosijn and Ingwersen [18] describe affective relevance as a cross-cutting factor, influencing all other relevance dimensions; and Saracevic [69] treats affective and motivational relevance as a single dimension. Certainly, state interest, in its role as a motivational variable, could function to amplify perceptions of topical, situational, and cognitive relevance and to influence the searcher's decision to select or reject documents.

Another approach is to frame interest as a component of "personal relevance" [13,32] - a notion that sidesteps the relationship between an information need and a document, and acknowledges that there can be direct relationships between people and documents that is not facilitated through conscious information needs. In sum, interest is clearly a factor in content preference and selection, and it would serve the broader research community if studies of interest make assumptions about the relationship between interest and relevance explicit.

\subsection{Interest and the user experience}

Understanding and improving the experience of searchers is an important goal in IIR. This has prompted research on the affective and experiential aspects of search, including the role of emotion and user engagement. Searcher interest is generally considered to be an important component of the search experience, although treatments vary substantially. Interest was examined in some of the first studies investigating the role of emotions in search; however, it was measured prior to the search session and treated as a feature of the searcher's task perception, rather than as an emotion in itself or a feature of the search experience $[29,44,45,60]$. The treatment of interest in work relating to user engagement $[20,49,52,56]$ and information experience $[28,74]$ has been more substantial and theory-driven. Within the user engagement framework, state interest is considered to be an attribute of engagement: an experience in which the attention and interest of a user are captured and maintained as they carry out an activity $[49,56]$. Motivation and interest triggered by features of the content, such as novelty, are considered to play a role in initiating user engagement [49] and the User Engagement Scale includes interest as a component of the Reward subscale in recognition of its motivating role [52]. Empirical studies have found that 
situational interest triggered by features of content ("interestingness") have been observed to foster user engagement in news consumption [5,55]. While close associations between interest and user engagement clearly exist, a full model of this relationship remains to be developed.

A related but distinct perspective on situational interest as a component of the "information experience" has been developed on the foundation of Silvia's [72] appraisal theory of interest [74,75]. Unlike most of the prior work in IIR, van der Sluis and colleagues clearly distinguish between individual interests - long term topical interests associated with familiarity [75]; and situational interest - automatic information responses associated with complexity and comprehensibility of the information presented to users [74]. Design-related contributions from this work consider textual features that facilitate interest $[28,74,75]$.

\subsection{Summary}

Notions of interest are deeply enmeshed in the core concepts of IIR research. Interest is understood to play a role in information needs, the assessment and selection of content as relevant, and the process and experience of searching. Despite this central positioning, interest has seldom been the direct focus of IIR research, and much of the work to date has failed to define the term or to differentiate between individual interests and the state of being interested. Foundational work on the psychology of interest has been scarce in treatments of interest with respect to information needs and relevance. Through recent studies that posit interest as a component of the user experience, a more complex picture is starting to emerge of the many relationships between interest and variables related to the search process (e.g., difficulty, familiarity, prior knowledge, task complexity, confidence) and information content (e.g., modality, textual complexity, sentiment, novelty and comprehensibility). However, without greater conceptual and theoretical clarity, it is difficult to make sense of these relationships and design studies capable of contributing to that goal.

\section{Manipulation and measurement of interest}

Moving beyond conceptual treatments of interest, we examined how it has been studied to date in IIR. We drew upon the Repository of Assigned Search Tasks, a collection of papers reporting on search studies that have employed ASTs [25]. A keyword search in RepAST for "interest" retrieves 86 results. A manual review of these results by the authors identified 33 papers that attempted to manipulate, control or measure interest. We expanded on the initial set of papers through searches in the ACM Digital Library and reference chaining to find 25 additional papers, for a total of 58 . We reviewed methods for manipulation and measurement of interest in these studies. While a small number of studies manipulated interest through system design or content selection [5,74], we focus on the dominant approach, which is through the use of ASTs. This process was not intended to be a systematic review, but allowed us to identify the range of approaches for studying interest in IIR.

\subsection{Task-based manipulation}

Search tasks are employed in many experimental user studies to motivate and control participants' interactions with the search system, and the realism of search tasks is critical for inducing authentic responses and valid data [11,16]. Researchers can approach this issue by using user-specified tasks, which are more authentic but less controlled, or by increasing the authenticity of ASTs. To this end, Borlund [10] proposed "simulated work task situations" (SWTS), which are widely, albeit inconsistently, used [11]. Among the requirements for SWTSs is that participants find them "topically interesting and/or of relevance to them" (p. 3). Borlund argues that if people are searching for their own purposes or interests, they are more likely to search realistically, as they are able to draw upon contextual knowledge. While valid, this association between interest and realism is not absolute, as many real-life searches are performed out of necessity or ignorance (i.e., Belkin's ASK [9]). This guideline for SWTSs helps to explain why many IIR studies aim to employ "interesting" tasks, although few provide an underlying rationale.

In the papers we reviewed, a range of approaches were used to manipulate interest through search tasks. We observed six patterns, and discuss each below, in light of the conceptual framing of interest mapped out in Section 2.

Assigning an interest: Some studies attempt to assign an individual interest to the searcher through the AST, as in the following example:

You have read that Alfred North Whitehead wrote a book, Science and Modern World, and you are interested in learning more in the concept of "simple location". You would like to learn about the various interpretations and views on Whitehead's simple location as a philosophical concept... [43].

While common, this approach is problematic because interest is an intrinsic motivator, which, by definition, cannot be assigned. Using this approach, many aspects of a genuine interest-driven search are likely to be absent, such as positive emotion, prior knowledge and intrinsic motivation. If the intent is to prompt an authentic and enthusiastic search session, it may be more effective to rely upon extrinsic motivation, for example, by framing the search task as a course assignment or requiring a post search report.

Partially-specified tasks: A long standing practice to increase the authenticity of laboratory user studies is to create AST templates in which participants fill in an area of personal interest. For example:

You plan to take a trip with (Participant decides who will come along and how many people there will be). The traveling season will be in [...], and you will go to (location)... [76].

This approach may increase the likelihood of state interest but there is a cost- benefit trade-off in terms of authenticity and experimental control. Indeed, Toms et al. [79] found no significant differences in behavioural or self-report measures between fully and partially specified ASTs in a web search study. The partially specified approach may be more valuable for in-depth, multisession or challenging tasks, such as the full-term research project that was the subject of Bowler's [14] study, where participants indicated that interest in their self-selected essay topic was key to their sustained motivation and success. 
Free search tasks: Open-ended ASTs are frequently used to prompt searches driven by individual or situational interest. For example, Klas et al. [37] had participants choose "an arbitrary task of current interest” (p. 481), and Burt and Liew [15] asked participants to "perform a short search on any topic of interest to them" (p. 285). This approach increases authenticity but leaves the researcher with little control, and therefore may be most appropriate for search scenarios that are, by their nature, hedonic or self-directed, and where interest is the primary driver, as in this fiction selection example:

You are in a library in a situation when you do not have a clear idea of what you would like to read. Please use the PIKI catalog to search for a novel of interest to you, which you would like to read.... [57].

In another example, the task context was specified, telling participants to gather news stories to share at a party, but left open at the search task level, suggesting they look for "interesting items" [51]. Findings showed that participants responded differently: some ignored the task context and selected items that triggered their interest, while others focused primarily on the sharing task to guide selection. This serves as a useful example of the difficulty of distinguishing interest effects from other motivating factors in studies using multi-layered task scenarios.

Real information needs: This approach is similar to free search tasks, but searchers are asked to bring one or more questions or information needs to the study session. Borlund and Pharo [13] report on a series of studies employing real information needs, the majority of which arose from "personal interests", such as hobbies and life responsibilities. This approach offers no control over search tasks, but is likely to prompt authentic search behaviour and may increase state interest. However, the notion of personal interest is broader than individual interest, as it includes life tasks that may not be intrinsically interesting.

In a useful example of how to balance control and authenticity, Poddar and Ruthven [60] used "genuine information needs" in combination with ASTs. While participants were not requested to bring interesting tasks per se, they reported being generally more interested and more knowledgeable of their genuine task than the assigned tasks. They also displayed more positive emotions while searching for their own task. Thus, it seems they naturally brought forward topics arising from individual interests and/or more readily entered into a state of interest for their own tasks. They also reported a greater sense of agency and ownership over their own tasks, which contributed to the more positive experience, suggesting that interest is only one of many possible differences between assigned and real search tasks.

Controlled task with choice or filter: Providing a list of possible ASTs and asking participants to select, rate, or rank them based on interest is an approach that has been used with many variations. In some studies, it is used to increase the interest level for participants, as recommended by Borlund [11]. $\mathrm{Xu}$ and Chen [83] offered four ASTs and participants chose the most interesting one, or were allowed to define their own topic. Others created two or more tasks per condition and allowed participants to choose the most interesting one for each [3,29]. In studies testing the differential effects of interest, it is important to ensure a range of interest levels. Edwards and Kelly [19] developed a set of eight search topics and asked participants to rank the topics in order from most to least interesting the day prior to taking part in the study. Participants were then assigned four ASTs using the two highest and two lowest ranked topics, and the manipulation was validated through pre-task self-report measures. This approach was effective, although post-task measures of interest should be included to measure state interest, in addition to a priori interest in the topic.

No choice but measured: Frequently, studies rely upon ratings or measurement of interest without allowing for choice or filtering. This is efficient, as all participants carry out all tasks; however, it cannot be relied upon to ensure a range of interest levels.

The nature of search tasks assigned in user studies can impact the level of state interest achieved. A number of techniques have been used for this purpose and these could be strengthened through more explicit attention to the underlying construct of interest in relation to the goals of individual studies. The design and use of search tasks to manipulate interest is closely tied to issues of measurement, which are addressed in the next section.

\subsection{Measurement of interest}

Valid measurement is critical to studies seeking to examine the role of interest as an independent variable or a dependent variable subject to experimental conditions. Notably, interest is subjective and dynamic, and can be expected to vary across a group of participants and search experiences, which adds to the challenge of measurement. The most common approach to measuring interest is through self-report data collected in pre- and/or post-task questionnaires $[43,44,55]$. This is appropriate, given the subjective nature of interest, but means that such studies are subject to the limitations of self-report data, such as tendencies to bias and variations in interpretation. In a typical approach, interest is measured on a scale using items such as, "How interesting do you find the topic of the search task?" [29]. Often, interest is measured together with a broader set of user perception variables, such as task difficulty, personal relevance, behavioural intentions to search or learn more, understandability, prior knowledge, etc. [e.g., 5, 19, 55]. In some cases, correlations among these measures are reported, showing that interest is intertwined with many features of the search experience. In addition, examining searchers' pre- and posttask interest can provide insight into how interest may have shifted over the course of the search session [50].

A limitation of this approach is that interest is rarely defined in the study or for participants, and care is not always taken to distinguish between interest in the topic, which may reflect a preexisting individual interest, and state interest triggered through interaction with content. If the intent is to capture the latter, posttask measures should be collected. However, the choice of pre- vs. post-task measurement of interest is seldom justified with respect to the type of interest under investigation.

Reliance upon self-report measures is consistent with research on interest in psychology [58]; however, in IIR, this is often handled through a single questionnaire item rather than a 
more robust approach using at least three items to reliably measure the concept [54]. One exception is [74], where state interest was measured as a construct comprised of three scale items, drawing upon the work of Silvia [72]. These included two semantic differentials: interesting-uninteresting and boringexciting, and a 7-point Likert scale: "I would be interested in reading more of this text". The UES [52], an established measure of user engagement, includes interest as a component of its "reward" dimension, and the Cognitive Absorption Scale has a related "curiosity" dimension [1]. Other work has associated interest with serendipity. McCay-Peet, Toms and Kelloway [46] developed a questionnaire based on four facets of serendipitous digital environments: highlights triggers, leads to the unexpected, enables connections and trigger-rich. Although the term "interest" is not used in the questionnaire, the trigger-rich" facet is described as, "the degree to which a digital environment contains a variety of information, ideas, or resources that is interesting and useful to the user" [46, p. 396]. These tools demonstrate that interest is associated with engaging, absorbing and serendipitous user experiences, but do not distinguish interest as a concept in its own right. More work in this vein is needed to develop standard tools for measuring interest in search.

Qualitative self-report data gathered through think-aloud protocols, interviews and questionnaires are used less frequently, but in a number of studies, these have led to valuable insights $[14,55,60,70]$. For example, in [55] participants were asked to elaborate on their ratings for interest and curiosity through an open-ended questionnaire. The study found that ratings reflected both individual interest/disinterest based on personal relevance and prior exposure, and situational interest prompted by factors such as novelty, emotional response, understandability and style of content. This suggests that including one question that asks searchers to rate their pre- and post-task interest may not capture the type of interest experienced by study participants, and that more open-ended elements could be used to check the manipulation of interest through ASTs and interpret other outcomes, such as search performance.

Other approaches to measuring interest include implicit measures, based on time on task and interaction data, such a reading time [17,34]. However, recent studies have raised questions about the validity of such measures with respect to interest, indicating that nuance in interpretation is required, as similar behaviours can result from divergent emotional states $[12,20]$. Similarly, interest is one source of motivation, but multiple sources of motivation may be present in a search study, which prompt similar behaviour (e.g., task and experimenter effects may motivate searchers to invest time and effort, even for uninteresting searches). Recent studies focused on state interest as an emotion and/or a component of the user experience have turned to physiological measures, such as heart rate, skin conductance and electromyography [20,53] and eye tracking [5]; and to neurological measures, such as EEG patterns [4]. This work is complex and is in early stages, both in IIR and in educational psychology, and holds promise as a means to disambiguate the effects of interest and other variables and deepen our understanding of the role of interest in information interaction.

\subsection{Summary}

Search tasks are commonly used to manipulate interest in IIR user studies. Of the six task patterns we identified, most draw informally upon the notion of individual interests, giving participants the opportunity to search for their own genuine needs, or to customize or select tasks based on these interests. Such tasks are likely to be effective in some contexts (e.g., hedonic search) but may be of limited value when more control is needed. For studies seeking to manipulate interest, offering participants a set of tasks and the opportunity to rank or rate them draws upon both individual interests and situational interest, and seems to be an effective approach to assigning tasks at different interest levels. A complementary approach would identify features of search tasks that are associated with situational interest, such as novelty, complexity and comprehensibility [72,74] and to vary these in the tasks provided. With respect to measurement, reliance upon simple self-report measures is the norm to date, with some limited efforts to develop more robust and multi-dimensional approaches, grounded in theory. It is hoped that this review will encourage researchers to build upon this work when designing future studies.

\section{Issues and Recommendations}

\subsection{Conceptual and theoretical clarity}

The lack of clarity on the concept of interest in IIR research has consequences. Previous work has rarely drawn on theories of interest, meaning that it has been defined and operationalized in inconsistent ways, making it difficult to trace the effects of interests across studies. It has been conflated with other variables (e.g., preference, selection, or choice [23]), which limits the validity of the findings. The everyday notion of interest, which is frequently used, fails to capture the construct of interest in all its complexities. We recommend that researchers adopt a theoretically-informed approach to the study of interest, as theory will serve as a guide for the development of hypotheses and in disentangling interest from other concepts, such as curiosity. Further, researchers should communicate the types of interest addressed in the work (e.g., disposition/state), and provide clear definitions and explicit information on how interest was manipulated, controlled and measured. This will allow future work to build on such studies. The body of theory on interest is not unitary, and includes distinct perspectives suited to diverse domains and research questions. We have outlined seminal work by Hidi and Renninger [30] and Silvia [72] and encourage researchers to draw upon these and other relevant theoretical framings.

\subsection{Interest as an explanatory variable}

Interest is a deeply ingrained feature of HII, but is likely to have a strong explanatory effect only for certain types of tasks and domains. These include searching as learning, self-directed, interestdriven search, and complex, challenging and lengthy tasks. In all of these cases, motivation, effort and positive affect associated with interest are likely to influence performance and outcomes. However, the manipulation of interest in such studies is a serious challenge due to the subjective nature of the appraisal mechanisms involved in 
activating state interest, which makes them difficult to control, and the idiosyncrasies of individual predispositions, which makes them challenging to predict. ASTs have been used for this purpose, although in most cases these have been designed to prompt high interest, in keeping with best practices [11], rather than to vary the level of interest. We need to move away from this approach in cases where we seek to study the differential effects of interest on the search process and outcomes.

Researchers may build on Edwards and Kelly's [19] approach in which study participants previewed and ranked ASTs based on interest; search task type was held constant to better isolate interest effects. This method could be extended by varying features or types of topics that are (un)likely to trigger situational interest. It is an open question if generic interest features of ASTs exist, which could be explored through crowdsourced studies of large sets of ASTs. Where possible, search content may also be systematically varied to affect participants' interest [e.g., 74]. While these methods may contribute to the manipulation of state interest, they are not guaranteed to produce consistent interest effects among participants and will need to be validated in each study.

\subsection{Interest as a confounding variable}

IIR studies often focus on variables that could be influenced by interest, such as task difficulty, prior knowledge, familiarity, satisfaction, and aspects of user engagement. In studies where the variable(s) of focus may be affected by interest, researchers may want to control for interest or measure interest and account for it when analyzing results. However, the nature of individual interests makes them difficult to predict and therefore difficult to control for. One way to deal with this may be to look at benchmarks within individuals, i.e., how much has the user's interest changed throughout the course of the study, to examine factors that may have enhanced or deterred interest. Alternatively, researchers could examine low, average and high interest relative to the context to explore the range of interest levels inherent in a study and the factors that may have contributed to lower or higher interest [55]. Thus, rather than aiming to control for interest, we can anticipate that it will mediate the user experience. Participants can be segmented based on interest level (low vs. high, increased vs. decreased interest): statistical methods (e.g., regression analysis) can be used to explore relationships amongst variables [50]) and qualitative approaches can aid in interpreting observations.

\subsection{Measuring interest}

Given the role of interest in so many aspects of IIR, it is important to measure it effectively. As noted in Section 4.2, while interest is frequently measured in IIR, simple (i.e., one statement) self-report approaches predominate. We lack standardized and validated tools, resulting in measures and instruments that vary substantially from one study to the next. Given the heavy use of self-report measures of interest in IIR, and, indeed, in psychology [58], we recommend, as a starting point, the development of one or more validated self-report scales for use in pre- and post-task questionnaires, addressing the different types (individual and state) and aspects of interest. There are some useful starting points in recent studies of interest (e.g., [74]) and such tools would contribute substantially to future research. To supplement quantitative studies, interviews may be useful, as these can reveal different understandings of the questionnaire items and capture unanticipated aspects of interest. Given that we are still learning about the impacts of interest, these kinds of insights will be generative.

Self-report data are essential to understand the subjective experience of interest, but are limited through reliance upon conscious experience and susceptibility to demand effects and recall biases [58]. As such, these should be complemented with other approaches that provide direct evidence of interest, such as implicit (behavioural), physiological and neurological measures [58,31]. However, such measures should be used with caution, on their own. Implicit measures do not capture the affective aspects of the search directly and are susceptible to confounds, notably between interest, frustration, difficulty [20,12]. Physiological and neurological measures are also subject to interpretation by researchers. Another limiting factor is the lack of clear baselines and benchmarks for the measurement of interest and related variables [41]. More work is needed to develop effective approaches to measure state interest. In the meantime, the use and reporting of multiple corroborating approaches is recommended.

\section{Conclusion}

Interest is a variable of importance in IIR. It is enmeshed in our understanding of information seeking, selection, and use, but is seldom examined on its own. This paper makes the case to do just that. By examining research on interest in a cognate discipline, educational psychology, we identify foundational concepts and theories that are relevant to IIR and map these onto the search process. We then explore how the notion of interest has been examined in IIR to date, identifying alignments and points of divergence in both the theory and the methodological approaches employed. We discuss issues and challenges inherent in the study of interest and make recommendations for moving forward. We encourage readers to consider the conceptual model outlined in this paper (Figure 2) as a framework for the development of research questions and hypotheses and space for future exploration. To support these efforts, more robust and consistent methods for the conceptualization and measurement of interest are needed. The wide applicability of interest in IIR means that the depth and scope of our coverage in this paper is limited. In the course of this work, we identified a number of studies that examine interest in a substantial way, and we hope that this work and the compiled list of references, can contribute to a future in depth review of the impact of interest on search and its relationships with other factors. Given the evidence from educational psychology, we see searching as learning as one of the most promising application spaces for interest research. Through greater conceptual alignment and respectful borrowing of theory, we hope to be able to strengthen IIR studies of interest in this area and others.

\section{ACKNOWLEDGMENTS}

We gratefully acknowledge the support of the Natural Science and Engineering Research Council (NSERC) funded Designing for People initiative at the University of British Columbia. 


\section{REFERENCES}

[1] Ritu Agarwal \& Elena Karahanna. 2000. Time flies when you're having fun: cognitive absorption and beliefs about information technology usage. MIS Q, 24 4 (Dec 2000), 665-694. http://www.jstor.org/stable/3250951

[2] Mary Ainley, Suzanne Hidi, and Dagmar Berndorff. 2002. Interest, learning, and the psychological processes that mediate their relationship. F. Educ. Psychol. 94, 3 (September 2002), 545-561. DOI: https://doi.org/10.1037/0022-0663.94.3.545

[3] Ioannis Arapakis, J. M. Jose, and P. D. G. Gray. 2008. Affective feedback: an investigation into the role of emotions in the information seeking process. In Proc. of the $31^{\text {st }}$ Annual International ACM SIGIR Conference (SIGIR '08), 395-402. DOI: https://doi.org/10.1145/1390334.1390403

[4] Ioannis Arapakis, Miguel Barreda-Ángeles, and Alexandre Pereda-Baños. 2019. Interest as a proxy of engagement in news reading: spectral and entropy analyses of EEG Activity Patterns. IEEE Trans. Affect. Comput. 10, 1 (January 2019), 100-114. DOI: https://doi.org/10.1109/TAFFC.2017.2682089

[5] Ioannis Arapakis, Mounia Lalmas, B. Cambazoglu, Mari-Carmen Marcos, and Joemon Jose. 2014. User engagement in online news: under the scope of sentiment, interest, affect, and gaze. 7. Assoc. Inf. Sci. Technol. 65, (October 2014). DOI: https://doi.org/10.1002/asi.23096

[6] Panos Balatsoukas and Ian Ruthven. 2010. What eyes can tell about the use of relevance criteria during predictive relevance judgment? In Proceedings of the third symposium on Information interaction in context (IIiX '10), Association for Computing Machinery, New York, NY, USA, 389-394. DOI https://doi.org/10.1145/1840784.1840844

[7] Albert Bandura. 1997. Self-efficacy: The Exercise of Control. W H Freeman/Times Books/ Henry Holt \& Co, New York, NY, US.

[8] Marcia J. Bates. 1989. The design of browsing and berrypicking techniques for the online search interface. Online Rev. 13, 5 (January 1989), 407-424. DOI: https://doi.org/10.1108/eb024320

[9] Nicholas J. Belkin (1980), Anomalous states of knowledge as a basis for information retrieval. Can. J. Inf. Sci., 5, 133-143.

[10] Pia Borlund. 2003. The concept of relevance in IR. F. Am. Soc. Inf. Sci. Technol. Hoboken 54, 10 (August 2003), 913-925.

[11] Pia Borlund. 2016. A study of the use of simulated work task situations in interactive information retrieval evaluations: a meta-evaluation. 7. Doc. 72, 3 (January 2016). DOI: https://doi.org/10.1108/JD-06-2015-0068

[12] Pia Borlund, Sabine Dreier, and Katriina Byström. 2012. What does time spent on searching indicate? In Proceedings of the 4th Information Interaction in Contex Symposium (IIIX '12), Association for Computing Machinery, New York, NY, USA, 184-193. DOI: https://doi.org/10.1145/2362724.2362756

[13] Pia Borlund and Nils Pharo. 2019. A need for information on information needs. Inf. Res. 24, 4 (2019). http:// http://informationr.net/ir/24-4/colis/colis1908.html

[14] Leanne Bowler. 2010. The self-regulation of curiosity and interest during the information search process of adolescent students. F. Am. Soc. Inf. Sci. Technol. 61, 7 (2010), 1332-1344. DOI: https://doi.org/10.1002/asi.21334

[15] Melissa Burt and Chern Li Liew. 2012. Searching with clustering: An investigation into the effects on users' search experience and satisfaction. Online Inf. Rev. 36, 2 (January 2012), 278-298. DOI: https://doi.org/10.1108/14684521211229075

[16] Katriina Byström and Preben Hansen. 2005. Conceptual framework for tasks in information studies. F. Am. Soc. Inf. Sci. Technol. 56, 10 (2005), 1050-1061. DOI: https://doi.org/10.1002/asi.20197

[17] Mark Claypool, Phong Le, Makoto Wased, and David Brown. 2001. Implicit interest indicators. In Proceedings of the 6th international conference on Intelligent user interfaces (IUI '01), ACM, New York, NY, USA, 33-40. DOI: https://doi.org/10.1145/359784.359836

[18] E. Cosijn and Peter Ingwersen. 2000. Dimensions of relevance. Inf. Process. Manag. 36, 4 (2000), 533-550.

[19] Ashlee Edwards and Diane Kelly. 2016. How does interest in a work task impact search behavior and engagement? In Proceedings of the 2016 ACM on Conference on Human Information Interaction and Retrieval (CHIIR '16), ACM, New York, NY, USA, 249-252. DOI: https://doi.org/10.1145/2854946.2855000

[20] Ashlee Edwards and Diane Kelly. 2017. Engaged or frustrated?: disambiguating emotional state in search. In Proceedings of the 40th International ACM SIGIR Conference on Research and Development in Information Retrieval (SIGIR '17), Association for Computing Machinery, New York, NY, USA, 125-134. DOI: https://doi.org/10.1145/3077136.3080818

[21] David Elsweiler, Max L. Wilson, and Lunn Brian Kirkegaard. 2011. Understanding casual-leisure information behaviour. In New Directions in Information Behaviour, Amanda Spink and Jannica Heinström (eds.). Emerald Group Publishing Limited, 211-241. DOI: https://doi.org/10.1108/S18760562(2011)002011a012

[22] Raya Fidel. 2012. Human Information Interaction: An Ecological Approach to Information Behavior, Raya Fidel, MIT Press (2012), p. 368, ISBN-13: 978-0-26201700-8

[23] Terri Flowerday, Gregory Schraw, and Joseph Stevens. 2004. The role of choice and interest in reader engagement. f. Exp. Educ. 72, 2 (2004), 93-114.
[24] Luanne Freund, Rick Kopak, and Heather O'Brien. 2016. The effects of textual environment on reading comprehension: Implications for searching as learning. 7. Inf. Sci. 42, 1 (February 2016), 79-93.

DOI: https://doi.org/10.1177/0165551515614472

[25] Luanne Freund and Barbara M. Wildemuth. 2014. Documenting and studying the use of assigned search tasks: RepAST. Proc. Am. Soc. Inf. Sci. Technol. 51, 1 (2014), 1-4. DOI: https://doi.org/10.1002/meet.2014.14505101122

[26] Sara M. Fulmer and Jan C. Frijters. 2011. Motivation during an excessively challenging reading task: the buffering role of relative topic interest. 7 . Exp. Educ 79, 2 (February 2011), 185-208. DOI: https://doi.org/10.1080/00220973.2010.481503

[27] Souvick Ghosh, Manasa Rath, and Chirag Shah. 2018. Searching as learning: exploring search behavior and learning outcomes in learning-related tasks. In Proceedings of the 2018 Conference on Human Information Interaction \& Retrieval (CHIIR '18), Association for Computing Machinery, New York, NY, USA, 22-31. DOI: https://doi.org/10.1145/3176349.3176386

[28] Richard Glassey and Leif Azzopardi. 2011. Finding interest in the stream. Proc. Am. Soc. Inf. Sci. Technol. 48, 1 (2011), 1-4. DOI: https://doi.org/10.1002/meet.2011.14504801297

[29] Jacek Gwizdka and Irene Lopatovska. 2009. The role of subjective factors in the information search process. F. Am. Soc. Inf. Sci. Technol. 60, 12 (2009), 2452-2464. DOI: https://doi.org/10.1002/asi.21183

[30] Suzanne Hidi and K. Ann Renninger. 2006. The Four-Phase Model of Interest Development. Educ. Psychol. 41, 2 (June 2006), 111-127. DOI: https://doi.org/10.1207/s15326985ep4102_4

[31] Suzanne Hidi and K. Ann Renninger. 2019. Interest development and its relation to curiosity: needed neuroscientific research. Educ. Psychol. Rev. 31, 4 (July 2019), 833-852.

[32] Peter Ingwersen and Kalervo Järvelin. 2005. The Turn: Integration of Information Seeking and Retrieval in Context. Springer, Cham, Switzerland.

[33] David H. Jonassen. 2000. Toward a design theory of problem solving. Educ Technol. Res. Dev. 48, 4 (December 2000), 63-85. DOI: https://doi.org/10.1007/BF02300500

[34] Kellar, M., Watters, C., Duffy, J., and Shepherd, M. 2004. Effects of time spent reading as an implicit measure of interest. In Proc. Am. Soc. Inf. Sci. Technol. 41 (2004). Providence, RI, 168-175.

[35] Soojung Kim and Dagobert Soergel. 2005. Selecting and measuring task characteristics as independent variables. Proc. Am. Soc. Inf. Sci. Technol. 42, 1 (2005). DOI: https://doi.org/10.1002/meet.14504201111

[36] Walter Kintsch. 1980. Learning from text, levels of comprehension, or: Why anyone would read a story anyway. Poetics 9, 1 (June 1980), 87-98. DOI: https://doi.org/10.1016/0304-422X(80)90013-3

[37] Claus-Peter Klas, Norbert Fuhr, and André Schaefer. 2004. Evaluating strategic support for information access in the DAFFODIL system. In Research and Advanced Technology for Digital Libraries (Lecture Notes in Computer Science), Springer, Berlin, Heidelberg, 476-487. DOI: https://doi.org/10.1007/978-3-54030230-8_43

[38] Andreas Krapp. 2000. Interest and human development during adolescence: an educational-psychological approach. In Motivational Psychology of Human Development. 109-128. DOI: https://doi.org/10.1016/S0166-4115(00)80008-4

[39] Andreas Krapp. 2002. An educational-psychological theory of interest and its relation to self-determination theory. In E. L. Deci \& R. M. Ryan (Eds.), Handbook of self-determination research (p. 405-427). University of Rochester Press.

[40] Andreas Krapp. 2007. An educational-psychological conceptualisation of interest. Int. F. Educ. Vocat. Guid. 7, 1 (April 2007), 5-21. DOI: https://doi.org/10.1007/s10775-007-9113-9

[41] Maria Lebow and Heather L. O'Brien. 2012. Is there a role for physiological methods in the evaluation of human-information interaction? Working Paper. DOI 10.14288/1.0107446 URI http://hdl.handle.net/2429/45315.

[42] Yuelin Li and Nicholas J. Belkin. 2008. A faceted approach to conceptualizing tasks in information seeking. Inf. Process. Manag. 44, 6 (November 2008), 18221837. DOI: https://doi.org/10.1016/j.ipm.2008.07.005

[43] Jingjing Liu, Chang Suk Kim, and Caitlin Creel. 2015. Exploring search task difficulty reasons in different task types and user knowledge groups. Inf. Process. Manag. 51, 3 (May 2015), 273-285.

DOI: https://doi.org/10.1016/j.ipm.2014.10.001

[44] Irene Lopatovska. 2009. Searching for good mood: examining relationships between search task and mood. Proc. Am. Soc. Inf. Sci. Technol. 46, 1 (2009), 1-13. DOI: https://doi.org/10.1002/meet.2009.1450460222

[45] Irene Lopatovska and Hartmut B. Mokros. 2008. Willingness to pay and experienced utility as measures of affective value of information objects: Users' accounts. Inf. Process. Manag. 44, 1 (January 2008), 92-104. DOI: https://doi.org/10.1016/j.ipm.2007.01.020

[46] Lori McCay-Peet, Elaine G. Toms and E. Kevin Kelloway. 2015. Examination of relationships among serendipity, the environment, and individual differences. Inf Process Manag, 51, 4, 391-412. https://doi.org/10.1016/j.ipm.2015.02.004

[47] Mark A. McDaniel, Paula J. Waddill, Kraig Finstad, and Tammy Bourg. 2000. The effects of text-based interest on attention and recall. f. Educ. Psychol. 92, 3 (September 2000), 492-502. DOI: https://doi.org/10.1037/0022-0663.92.3.492 
[48] Austin Lee Nichols and Jon K. Maner. 2008. The good-subject effect: investigating participant demand characteristics. 7. Gen. Psychol. 135, 2 (April 2008), 151-166. DOI: https://doi.org/10.3200/GENP.135.2.151-166

[49] Heather O'Brien. 2016. Theoretical perspectives on user engagement. In Why Engagement Matters: Cross-Disciplinary Perspectives of User Engagement in Digital Media, Heather O'Brien and Paul Cairns (eds.). Springer International Publishing, Cham, 1-26. DOI: https://doi.org/10.1007/978-3-319-27446-1_1

[50] Heather L. O'Brien, Jaime Arguello and Rob Capra. 2020. An empirical study of interest, task complexity, and search behaviour on user engagement. Inf Process Manag, 57, 3 (May, 2020). https://doi.org/10.1016/j.ipm.2020.102226

[51] Heather O'Brien, Luanne Freund, and Stina Westman. 2014. What motivates the online news browser? News item selection in a social information seeking scenario. Inf. Res. Int. Electron. F. 19, 3 (2014). Retrieved October 11, 2020 from https://research.aalto.fi/en/publications/what-motivates-the-online-newsbrowsernews-item-selection-in-a-so

[52] Heather L. O’Brien, Paul Cairns, and Mark Hall. 2018. A practical approach to measuring user engagement with the refined user engagement scale (UES) and new UES short form. Int. F. Hum.-Comput. Stud. 112, (April 2018), 28-39. DOI: https://doi.org/10.1016/j.ijhcs.2018.01.004

[53] Heather L. O'Brien and Mahria Lebow. 2013. Mixed-methods approach to measuring user experience in online news interactions. 7. Am. Soc. Inf. Sci. Technol. 64, 8 (2013), 1543-1556. DOI: https://doi.org/10.1002/asi.22871

[54] Heather L. O'Brien and Lori McCay-Peet. 2017. Asking "good" questions: questionnaire design and analysis in interactive information retrieval research. In Proc. of the 2017 Conference on Human Information Interaction and Retrieval. (CHIIR '17), ACM, New York, NY, USA, 27-36. DOI https://doi.org/10.1145/3020165.3020167

[55] Heather L. O'Brien and Jocelyn McKay. 2016. What makes online news interesting? Personal and situational interest and the effect on behavioral intentions. Proc. Assoc. Inf. Sci. Technol. 53, 1 (2016), 1-6. DOI: https://doi.org/10.1002/pra2.2016.14505301150

[56] Heather L. O'Brien and Elaine G. Toms. 2008. What is user engagement? A conceptual framework for defining user engagement with technology. 7. Am. Soc Inf. Sci. Technol. 59, 6 (April 2008), 938-955. DOI: https://doi.org/10.1002/asi.20801

[57] Suvi Oksanen and Pertti Vakkari. 2012. In search of a good novel, neither reading activity nor querying matter, but examining search results does. In Proc. of the 4th Information Interaction in Context Symposium (IIIX '12), Association for Computing Machinery, New York, NY, USA, 12-20. DOI: https://doi.org/10.1145/2362724.2362731

[58] Reinhard Pekrun. 2019. The murky distinction between curiosity and interest: state of the art and future prospects. Educ. Psychol. Rev. 31, 4 (December 2019), 905-914. DOI: https://doi.org/10.1007/s10648-019-09512-1

[59] Peter L. T. Pirolli. 2007. Information Foraging Theory: Adaptive Interaction with Information (1st ed.). Oxford University Press, Inc., USA.

[60] Arti Poddar and Ian Ruthven. 2010. The emotional impact of search tasks. In Proc. of the third symposium on Information interaction in context (IIIX '10), Association for Computing Machinery, New York, NY, USA, 35-44. DOI: https://doi.org/10.1145/1840784.1840792

[61] K. Ann Renninger and Suzanne Hidi. 2015. The Power of Interest for Motivation and Engagement. Routledge. DOI: https://doi.org/10.4324/9781315771045

[62] K. Ann Renninger, Suzanne Hidi, and Andreas Krapp, eds. 2014. The Role of interest in Learning and Development. Psychology Press. DOI: https://doi.org/10.4324/9781315807430

[63] K. Ann Renninger and Robert H. Wozniak. 1985. Effect of interest on attentional shift, recognition, and recall in young children. Dev. Psychol. 21, 4 (July 1985), 624-632. DOI: https://doi.org/10.1037/0012-1649.21.4.624

[64] Ashley A. Rowland, Eva Knekta, Sarah Eddy, and Lisa A. Corwin. 2019. Defining and measuring students' interest in biology: an analysis of the biology education literature. CBE Life Sci. Educ. 18, 3 (2019). DOI: https://doi.org/10.1187/cbe.19-020037

[65] Ian Ruthven. 2019. Making meaning: a focus for information interactions research. In Proc. of the 2019 Conference on Human Information Interaction and Retrieval (CHIIR '19), Association for Computing Machinery, New York, NY, USA, 163-171. DOI: https://doi.org/10.1145/3295750.3298938

[66] Ian Ruthven, Mark Baillie, and David Elsweiler. 2007. The relative effects of knowledge, interest and confidence in assessing relevance. f. Doc. 63, 4 (January 2007), 482-504. DOI: https://doi.org/10.1108/00220410710758986
[67] R. M. Ryan and E. L. Deci. 2000. Self-determination theory and the facilitation of intrinsic motivation, social development, and well-being. Am. Psychol. 55, (2000), 68-78. DOI: https://doi.org/10.1037/0003-066X.55.1.68

[68] Carol Sansone, Danielle M. Geerling, Dustin B. Thoman, and Jessi L. Smith. 2019 Self-regulation of motivation: a renewable resource for learning. In The Cambridge Handbook of Motivation and Learning, K. Ann Renninger and Suzanne E. Hidi (eds.). Cambridge University Press, Cambridge, 87-110. DOI: https://doi.org/10.1017/9781316823279.006

[69] Tefko Saracevic. 1997. The stratified model of information retrieval interaction: extension and applications. Proc. ASIS Annual. Meet. 34, (1997), 313-27.

[70] Reijo Savolainen and Jarkko Kari. 2006. User-defined relevance criteria in web searching. f. Doc. 62, 6 (January 2006), 685-707. DOI: https://doi.org/10.1108/00220410610714921

[71] Klaus R. Scherer, Angela Schorr, and Tom Johnstone. 2001. Appraisal Processes in Emotion: Theory, Methods, Research. Oxford University Press.

[72] Paul J. Silvia. 2008. Interest-the curious emotion. Curr. Dir. Psychol. Sci. 17, 1 (February 2008), 57-60. DOI: https://doi.org/10.1111/j.14678721.2008.00548

[73] Frans van der Sluis. 2013. When Complexity becomes Interesting: An Inquiry into the Information eXperience. (August 2013). DOI: https://doi.org/10.3990/ 1.9789036505673

[74] Frans van der Sluis, Egon L. van den Broek, Richard J. Glassey, Elisabeth M. A. G. van Dijk, and Franciska M. G. de Jong. 2014. When complexity becomes interesting. 7. Assoc. Inf. Sci. Technol. 65, 7 (2014), 1478-1500. DOI: https://doi.org/10.1002/asi.23095

[75] Frans van der Sluis, Richard J. Glassey, and Egon L. van den Broek. 2012. Making the news interesting: understanding the relationship between familiarity and interest. In Proc. of the 4th Information Interaction in Context Symposium (IIIX '12), Association for Computing Machinery, New York, NY, USA, 314-317. DOI https://doi.org/10.1145/2362724.2362783

[76] Hitoshi Terai, Hitomi Saito, Yuka Egusa, Masao Takaku, Makiko Miwa, and Noriko Kando. 2008. Differences between informational and transactional tasks in information seeking on the web. In Proc. of the second international symposium on Information interaction in context (IIiX '08), Association for Computing Machinery, New York, NY, USA, 152-159. DOI: https://doi.org/10.1145/1414694.1414728

[77] Dustin B. Thoman, Jessi L. Smith, and Paul J. Silvia. 2011. The resource replenishment function of interest. Soc. Psychol. Personal. Sci. 2, 6 (November 2011), 592-599. DOI: https://doi.org/10.1177/1948550611402521

[78] Elaine G. Toms. 2000. Understanding and facilitating the browsing of electronic text. Int. 7. Human-Computer Studies, 52, 3, 423-452. DOI: 10.1006/ijhc.1999.0345

[79] Elaine G. Toms, Richard Kopak, Joan Bartlett, Luanne Freund, J. Heaton, and A. Olsen. 2001. Selecting versus describing: the efficacy of categories in exploring the Web. In Proceedings of the 10th Text Retrieval Conference, Gaithersburg, MA.

[80] Ryen W. White and Resa A. Roth. 2009. Exploratory Search: Beyond the QueryResponse Paradigm. Morgan Claypool Publishers. DOI: https://doi.org/10.2200/S00174ED1V01Y200901ICR003

[81] Allan Wigfield and Jacquelynne S. Eccles. 2002. The development of competence beliefs, expectancies for success, and achievement values from childhood through adolescence. In Development of Achievement Motivation, Allan Wigfield and Jacquelynne S. Eccles (eds.). Academic Press, San Diego, 91-120. DOI https://doi.org/10.1016/B978-012750053-9/50006-1

[82] Barbara M. Wildemuth and Luanne Freund. 2012. Assigning search tasks designed to elicit exploratory search behaviors. In Proc. of the Symposium on Human-Computer Interaction and Information Retrieval (HCIR '12), ACM, New York, NY, USA, 4:1-4:10. DOI: https://doi.org/10.1145/2391224.2391228

[83] Yunjie (Calvin) Xu and Zhiwei Chen. 2006. Relevance judgment: What do information users consider beyond topicality? 7. Am. Soc. Inf. Sci. Technol. 57, 7 (2006), 961-973. DOI: https://doi.org/10.1002/asi.20361

[84] Yinglong Zhang, Rob Capra, and Yuan Li. 2020. An in-situ study of information needs in design-related creative projects. In Proc. of the 2020 Conference on Human Information Interaction and Retrieval (CHIIR '20), Association for Computing Machinery, New York, NY, USA, 113-123. DOI: https://doi.org/10.1145/3343413.3377973 